\title{
Multipartite purification, multiboundary wormholes, and islands in $\mathrm{AdS}_{3} / \mathrm{CFT}_{2}$
}

\author{
Aranya Bhattacharya $\odot^{*}$ \\ Saha Institute of Nuclear Physics 1/AF Bidhannagar, Calcutta 700064, India \\ and Homi Bhabha National Institute Training School Complex, Anushakti Nagar, Mumbai 400085, India
}

(Received 26 May 2020; accepted 29 July 2020; published 17 August 2020)

\begin{abstract}
The holographic duals of entanglement of purification (EoP) through the entanglement wedge cross section (EWCS) have been a well-discussed topic in the literature recently. More general entanglement measures involving multipartite information and their holographic duals have also been proposed. On the other hand, the recent program reproducing the Page curve in black hole entropy with the notion of islands has also been an intriguing area of interest. A toy model involving multiboundary wormholes in $\mathrm{AdS}_{3}$ was able to capture many interesting facts about such calculations. In such a toy model, the notion of islands was intuitively connected to quantum error correction. We try to bridge the ideas of the two programs, especially in $\mathrm{AdS}_{3} / \mathrm{CFT}_{2}$, and give a description of the islands in terms of multipartite entanglement of purification. This clarifies a few simplified assumptions made while describing the toy model and also enables us to understand the familiar information paradox within the framework of the same model.
\end{abstract}

DOI: 10.1103/PhysRevD.102.046013

\section{INTRODUCTION}

In the last several decades, the black hole information [1] problem has resurfaced more often than not in studies of $\mathrm{BH}$ thermodynamics as well as in studies of black holes through holography. Holography has proved to be a very insightful candidate in many such studies. The way to tackle the information problem in holography has been boosted by quantum information-theoretic studies [2-9] done in holography, where the stand-out contribution comes from the seminal Ryu-Takayanagi [10-12] conjecture relating bulk codimension-two minimal surfaces to boundary entanglement entropy.

Recently, in [13], the authors conjectured bulk counterparts of more general information-theoretic quantities like the entanglement of purification. This has been studied both in gravitational setups and in free field theories [14-17]. In the gravitational ones, the holographic EoP is conjectured to be related to the entanglement wedge cross section. Such results include the study of pure AdS, BTZ black holes, as well as time-dependent scenarios. Studies of a few other quantum information-theoretic quantities-e.g., multipartite entanglement of purification

\footnotetext{
*aranya.bhattacharya@saha.ac.in
}

Published by the American Physical Society under the terms of the Creative Commons Attribution 4.0 International license. Further distribution of this work must maintain attribution to the author(s) and the published article's title, journal citation, and DOI. Funded by SCOAP.
[18-22] and reflected entropy [23-28]-have been motivated by the EoP computations.

On the other hand, in the last year or so, the information paradox problem has gone through an intriguing turn of events due to several interesting papers [29-33], where the authors try to describe the famous Page curve using the quantum, corrected holographic entanglement entropy for evaporating black hole geometries (note that these are timedependent scenarios). The motivation for these works is also somewhat related to the entanglement wedge [34] studies done both in holographic and in nonholographic setups (using quantum maximin surfaces)[35]. The results have attracted a lot of interest since they have been able to explain the long-lasting problem of convincingly describing the Page curve.

Surprisingly, the two above-mentioned studies have yet to be discussed together. The motivation and goal of this paper is to point out the similarities in the study of the EWCS (conjectured EoP) and the Page curve for black holes.

In this paper, we discuss the possible connections between the two studies in the case of $\mathrm{AdS}_{3}$. We use the concepts of the holographic dual of multipartite entanglement of purification for states in the boundary of pure $\mathrm{AdS}_{3}$. We use these concepts in the toy model of an evaporating black hole [33], where the evaporating black hole is initially treated as a big black hole, whereas the emitted Hawking quanta are realized by smaller black holes in $\mathrm{AdS}_{3}$. All of the black holes are connected by a multiboundary wormhole (MBW). An important part of this connection is the realization of MBWs in $\mathrm{AdS}_{3}$ as quotients of pure $\mathrm{AdS}_{3}$, where the boundaries are identified 
by removing semicircles and introducing orientationreversing isometries in a time slice of $\mathrm{AdS}_{3}$.

The CFT at each of the boundaries is a subregion of the CFT that we deal with while discussing multipartite entanglement of purification, and there, the horizon lengths are independent of each other. Therefore, we can tune the size of the black holes (Hawking quanta would be smaller black holes compared to the one that is emitting them) by choosing the parameters (or intervals in the boundary CFT of pure $\mathrm{AdS}_{3}$ ) and fixing them in such a way that the smaller black holes would be of comparable sizes. The bigger black hole keeps decreasing in size while creating more and more smaller black holes, which increases the exits of the wormhole. In the picture described in the toy model, the preferred HRT (Hubeny-Rangamani-Takayanagi) surface changes over time with the inclusion of a shared interior. This shared interior is conjectured to be the analog of the nontrivial islands mentioned in Maldacena's papers.

We try to get an understanding of the islands from the perspective of multipartite EoP. We make comments on the shared interior from the point of view of quantum error connection $[34,36,37]$ but by taking a detour through entanglement of purification. This is not very surprising since both of the programs are heavily dependent on the ideas of entanglement wedge reconstruction and nesting. Our comments relate the body of a multiboundary wormhole to a geometric pure state construction. We determine how, in such a model, there are two sides to the story. The classical picture gives us an intuitive understanding of the islands and helps us reproduce the Page curve. But the quantum version of the extremal surface again gives the familiar paradox addressed by Hawking. Finally, we give resolutions for understanding how to deal with the problem both in the toy model and in the entanglement of purification case.

The rest of the paper is organized as follows. We give a brief review of the purification program in Sec. II. In Sec. III, we review the basic ideas that have been instrumental in the derivation of the Page curve for black holes and Hawking radiation. We also discuss the toy model which motivated this work. We discuss the multiboundary wormhole constructions in $\mathrm{AdS}_{3}$ by quotienting $\mathrm{AdS}_{3}$ with several pictures that describe the situation in a simple way. We find that it is actually necessary to review the two topics in such a manner as it helps us obtain the connections made in the later half. In Sec. IV, we compare the two programs to point out the similarities and differences and find how the connections can feed each other in several interesting ways. Finally, in Sec. V, we discuss resolutions to strengthen the connection, and give a few open problems and future directions that one can pursue.

\section{NOTE ON ENTANGLEMENT OF PURIFICATION}

Purification is the process of making a mixed state pure. There are numerous measures in quantum information theory, most of which are sensitive to the state at hand being a pure one. A pure state is a state for which one can use the standard ket $(|\psi\rangle)$ notation of quantum mechanics. The density matrix of a pure state $\left(\rho_{\text {pure }}=|\psi\rangle\langle\psi|\right)$ follows a simple relation,

$$
\operatorname{Tr}\left[\rho_{\text {pure }}\right]=\operatorname{Tr}\left[\rho_{\text {pure }}^{2}\right]=1 .
$$

A mixed state has many descriptions from the perspective of quantum mechanics, the most famous one being the following: A mixed state is a classical probabilistic mixture of all the possible outcome states. It only has a representation in terms of the density matrix $\left(\rho_{\text {mixed }}=\sum_{i} p_{i}\left|\psi_{i}\right\rangle\left\langle\psi_{i}\right|\right)$ and does not have a simple ket description,

$$
\operatorname{Tr}\left[\rho_{\text {mixed }}\right]=1, \quad \operatorname{Tr}\left[\rho_{\text {mixed }}^{2}\right]<1 .
$$

The standard way to purify a mixed state is to add an auxiliary system with the mixed state where the total state, after adding the auxiliary system, becomes a pure state and the mixed state becomes a particular reduced state after tracing out a few degrees of freedom from the purified state. However, for a single mixed state, there might exist more than one way of purification. A particular one is chosen with respect to the information-theoretic measure one wants to calculate in a given scenario.

\section{A. Definitions and properties}

Entanglement of purification, as the name suggests, is related to purification of a mixed quantum state. The precise definition of entanglement of purification between $A$ and $B$ for a bipartite mixed state $A B(=A \cup B)$ is the minimal entanglement entropy between $A A^{\prime}$ and $B B^{\prime}$, where $A^{\prime}$ and $B^{\prime}$ are auxiliary systems added to make the whole state $A A^{\prime} B B^{\prime}$ pure (see Fig. 1).

Similarly, multipartite entanglement of purification is where, instead of a bipartite mixed state $A B$, we start with a multipartite mixed state $A_{1} A_{2} \ldots A_{n}$ and add auxiliary systems $A_{1}^{\prime}, A_{2}^{\prime}, \ldots, A_{n}^{\prime}$ to make it pure and then compute the minima of the sum over all $S_{A_{i} A_{i}^{\prime}}$ for $i=1, \ldots, n$.

The mathematical expression is written as follows.

\begin{tabular}{|c|c|}
\hline$A$ & $B$ \\
\hline$A^{\prime}$ & $B^{\prime}$ \\
\hline
\end{tabular}

FIG. 1. Schematic diagram of bipartite purification $\left(A A^{\prime} B B^{\prime}\right.$ forms a pure state and EoP is the entanglement between $A A^{\prime}$ and $\left.B B^{\prime}\right)$. 
Definition.-For an $n$-partite mixed state, with density matrix $\rho_{A_{1} A_{2} \ldots A_{n}}$, the multipartite entanglement of purification is defined as

$$
\Delta_{n(P)}\left(\rho_{A_{1} A_{2} \ldots \ldots A_{n}}\right)=\min _{|\psi\rangle_{A_{1} A_{1}^{\prime} A_{2} A_{2}^{\prime} \ldots \ldots A_{n} A_{n}^{\prime}}} \sum_{i=1}^{n} S_{A_{i} A_{i}^{\prime}} .
$$

This boils down to the definition of bipartite entanglement of purification once $n$ is taken to be 2 with appropriate normalization ( $\frac{1}{n}$ factor in the above definition). Of course, for the bipartite case, the EoP is symmetric under the two parts $\left(S_{A A^{\prime}}=S_{B B^{\prime}}\right)$. Let us call the bipartite EoP as $E_{P}\left(=\Delta_{2(P)}\right)$.

Properties.-The properties are as follows:

(1) If one of the systems gets decoupled, $\rho_{A_{1} \ldots A_{n}}=$ $\rho_{A_{1} \ldots A_{n-1}} \otimes \rho_{A_{n}}$, and then

$$
\Delta_{P}\left(A_{1}: \ldots: A_{n}\right)=\Delta_{P}\left(A_{1}: \ldots: A_{n-1}\right) .
$$

(2) For an $n$-partite pure state $|\psi\rangle_{A_{1} \ldots A_{n}}$,

$$
\Delta_{P}\left(A_{1}: \ldots: A_{n}\right)=\sum_{i=1}^{n} S_{A_{i}} .
$$

(3) For an $n$-partite product state $\left(\rho_{A_{1} \ldots A_{n}}=\right.$ $\left.\rho_{A_{1}} \otimes \rho_{A_{2}} \otimes \ldots \otimes \rho_{A_{n}}\right)$,

$$
\Delta_{P}\left(A_{1}: \ldots: A_{n}\right)=0 .
$$

(4) $\Delta_{p}$ is bounded from above as follows:

$$
\begin{aligned}
& \Delta_{P}\left(A_{1}: \ldots: A_{n}\right) \\
& \quad \leq \min _{i}\left(S_{A_{1}}+\ldots+S_{A_{1} \ldots A_{i-1} A_{i+1} \ldots A_{n}}+\ldots S_{A_{n}}\right) .
\end{aligned}
$$

(5) $\Delta_{p}$ is bounded from below as follows:

$$
\Delta_{P}\left(A_{1}: \ldots: A_{n}\right) \geq I\left(A_{1}: \ldots: A_{n}\right),
$$

where $I\left(A_{1}: \ldots: A_{n}\right)$ is the $n$-partite mutual information.

All of these are followed by bipartite EoP if one takes $n=2$.

\section{B. Holographic duals}

The holographic duals of various purification measures have been proposed in Refs. [13,19,38,39]. Here, we first discuss the holographic dual $\left(E_{W}\right)$ of the bipartite $\operatorname{EoP}\left(E_{P}\right)$ and then the $n$-partite case. For holographic states, it was conjectured that the holographic dual of $E_{P}$ is the minimum entanglement wedge cross section, which is the dotted line in Fig. 2. The mathematical definition of the entanglement wedge cross section is the following: $E_{W}(A: B)=$ $\min \left\{\operatorname{Area}(\Gamma) ; \Gamma \subset M_{A B}-M_{A \cap B}\right\}$ separates $A \backslash B$ and $B \backslash A$, where $M$ denotes the entanglement wedge of some

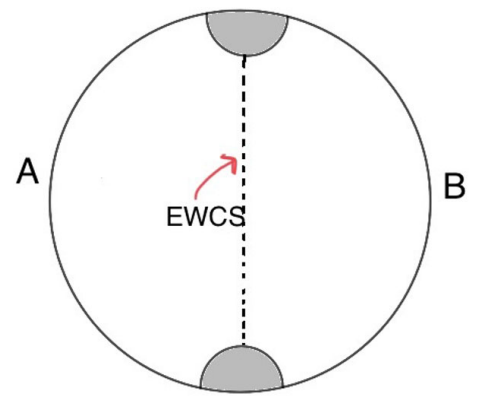

FIG. 2. Entanglement wedge cross section (holographic dual of bipartite entanglement of purification).

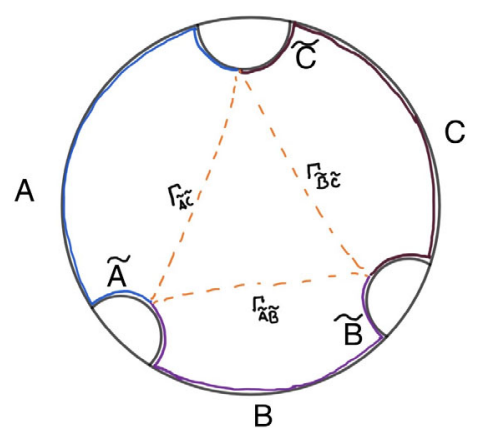

FIG. 3. EWCS for tripartite EoP: $A, B, C$ and the HRT geodesics are combined as $\tilde{A} \tilde{B} \tilde{C}$ and considered to form a tripartite geometric pure state.

specified interval in the boundary CFT. Note that $A / B=$ $(A-A \cap B)$ and $B / A=(B-B \cap A)[13,18,19]$.

For multipartite states, one needs to consider subregions involving boundary and bulk subregions to redefine $\tilde{A}, \tilde{B}, \tilde{C}$ (for a tripartite case) and then compute the multipartite minimal entanglement wedge cross section $\Gamma_{\tilde{A} \tilde{B} \tilde{C}}$, where $\tilde{A} \tilde{B} \tilde{C}$ is a geometric pure state. This is pictorially given in Fig. $3[19,40]$. Actually, in the case of bipartite entanglement of purification, the boundary subregion $A \cup B$ is typically a mixed state, but $\left(A \cup B \cup H R T_{1} \cup H R T_{2}\right)$ is considered to be a geometric pure state and the minimal length dividing the whole system into two is considered to be the bipartite entanglement of purification. The HRTs serve as the ancilla systems ( $A_{i}^{\prime}$ parts mentioned in Sec. II A), which are added to make the geometric state a pure state.

In [13], it has been checked that $E_{W}$ follows the same set of properties as $E_{P}$, whereas, in [19], $\Delta_{n(W)}$ and $\Delta_{n(P)}$ have been found to share the same set of properties.

\section{NOTE ON PAGE CURVE STUDY AND TOY MODEL}

This is a very brief review of the recent program [29-33] that has been instrumental in describing the time evolution of a black hole to be a unitary process by considering the combination of an evaporating black hole and the Hawking 
radiation to form a pure state. It was then shown that using particular techniques, each system's entanglement entropy follows the same curve, which is not continuously growing but indeed decreases after the Page time. This is a pathbreaking result since it is the very first time that a program has been able to arguably solve the long-standing information paradox. Although it has been argued since the discovery of AdS/CFT that it can solve the information paradox, this is the very first concrete example that shows it in a somewhat convincing manner.

We have yet to make complete sense of the techniques that made this program successful in handling the task. It involves the introduction of certain bulk regions called islands, which are essential to derive a Page curve for the emitted Hawking radiation, along with new but familiar concepts of the quantum extremal surface. In the following subsections, we first describe the basic ideas of the computations. We then discuss a simple toy model involving a multiboundary wormhole which successfully reproduces the Page curve and gives us an interesting and intuitive understanding of the actual computations just by considering classical RT surfaces instead of quantum extremal surfaces.

\section{A. Information paradox and resolutions (islands)}

If there is information flow between two parts of a system, then the information missing in one part should necessarily show up in the other part. But, in the case of black holes, this scenario has been a long-standing problem. In the case of black holes, the way one typically compares information inside and outside the black holes is by specifying the entanglement between the two systems. The paradox appearing in this computation is peculiar since the information found in the radiation outside the black hole seems to be more than what the black hole can store. The entanglement entropy, which is a standard information measure between two entangled states, of the radiation outside the black hole is found to be growing for a very long time, whereas the black hole's entropy seems to decrease over time. They cross each other long before the radiation entropy saturates. But, since the evaporating black hole and the radiation states should form a combination, which is a pure state, the entanglement between them should always be the same. This is one of many ways in which the information paradox can be realized. Let us explain this method a bit more concretely.

We assume that the radiation state is just a combination of the Hawking quanta radiated by an evaporating black hole. For all such Hawking quanta, there is a partner quanta behind the black hole horizon, which is entangled with its outside partner. Let us schematically write the radiation quantas and their partner modes as a combination, which is a pure state,

$$
|\psi\rangle_{\mathrm{rad}} \equiv \sum_{\omega, n} e^{-\frac{\omega n}{2}}|n\rangle_{\mathrm{in}}|n\rangle_{\mathrm{out}},
$$

at any point in time $t$, where the time keeps track of the frequencies $\omega$ (summed over) and $n$ counts the entangled pairs of Hawking quanta emitted till that time.

Now, tracing over $|n\rangle_{\text {in }}$ states, one can find the reduced density matrix of the out state $\left(\rho_{\text {rad,out }}\right)$, which, in this case, is in the form of a thermal density matrix,

$$
\rho_{\text {rad,out }} \equiv \sum_{\omega, n} e^{-\omega n}|n\rangle_{\text {out }}\left\langle\left. n\right|_{\text {out }} .\right.
$$

This leads to the paradox since, once the entanglement entropy is calculated for this reduced density matrix following the usual formula of von Neumann entropy, it keeps growing until the black hole evaporates ( $n$ increases). On the other hand, the Bekenstein-Hawking entropy, which is supposed to be representative of black hole entropy, keeps decreasing as the black hole evaporates and the area decreases. After some time (known as the Page time),

$$
S\left(\rho_{\text {rad,out }}\right)>\frac{A_{\mathrm{BH}}}{4 G} .
$$

The situation then becomes more complicated as the bipartite state between the radiation outside and the black hole becomes much more entangled than the maximum entanglement the black hole can ever manage microscopically. The black hole apparently seem to have more entanglement than microstates available. This is a contradiction.

There have been several attempts to solve this paradox and derive a formula which follows the well-known Page evolution (grows initially, then decreases to zero after the Page time). But, until very recently, there has not been a convincing way to do this. References [29-33] introduce certain regions, called islands, which are null before the Page time but are non-null (behind the black hole horizon) after the Page time. This process also has to be considered for computing the entanglement entropy of the radiation states. Given such a situation, one also has to work with the idea of quantum extremal surfaces since, while computing the entanglement associated with the nontrivial regions behind the horizon, Ryu-Takayanagi also contributes, in a nontrivial way, to the entanglement entropy of the radiation states.

Figure 4 gives us a better understanding. We consider a two-sided $\mathrm{BH}$ in $\mathrm{AdS}_{3}$. The extreme left and extreme right regions represent nongravitational flat space (NGF) coupled with the asymptotic AdS boundaries. These are needed as we are considering evaporating black holes, and the coupled NGFs provide a way to introduce absorbing boundary conditions in the shared boundary through which outside Hawking quanta can escape (unlike the eternal BH case, where the outside quantas are reflected from the AdS boundary to feed the black hole). Using this method, we compute the entanglement entropy of the outside quantas in the NGFs. This is like stacking up the quantas escaping 


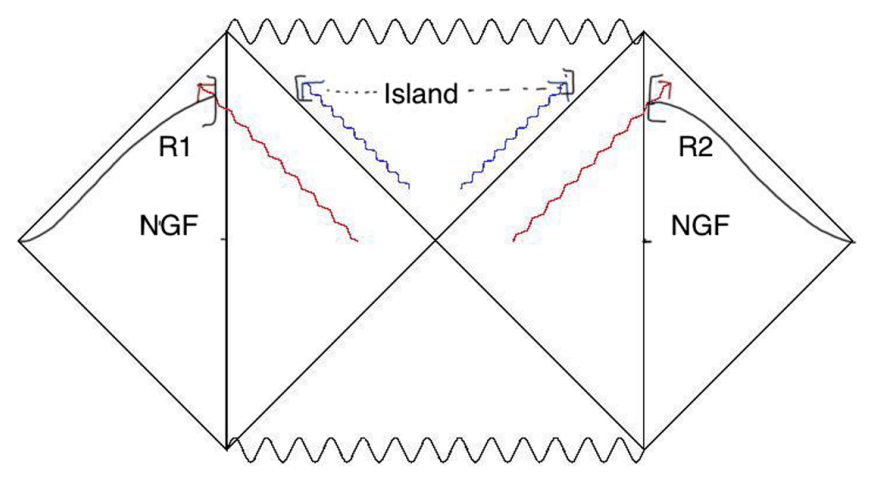

FIG. 4. Penrose diagram of two sided Black Hole with nontrivial island included (red and blue lines represent Hawking partner modes outside and inside the black hole horizon respectively.)

AdS in the NGFs. But this would again lead to the usual paradox. Say we compute entanglement entropy at an anchored time slice $t$ for a region from infinity (in the NGF) to very near the AdS boundary on both sides of AdS. Let us call these two regions $R 1$ and $R 2$ and their union $(R 1 \cup R 2=) R$. Here, $S_{\text {out }}[R]$ would again grow for a very large time and lead to the information loss.

The introduction of the islands is useful here, along with the consideration of quantum extremal surfaces. When islands are included (shaded region behind the horizon, after the Page time), the new notion of entanglement entropy for the outside quantas is

$S_{\text {out }}[R]($ new $)=\min _{I}\left[\operatorname{ext}_{I}\left\{\frac{A(\partial I)}{4 G}+S_{\text {usual }}[R \cup I]\right\}\right]$,

where $\partial I$ is the boundary of the region enclosed by the islands.

For a given timescale, first one has to take all choices of I (any interval in $\mathrm{AdS}_{3}$, inside or outside the horizon can be a candidate for I). Then, the sum of the items in curly brackets has to be extremized. The notion is that, in general, there exist more than one choice of I for which the sum is extremized. One has to choose the one which minimizes the sum at any given time. This solves the paradox since one finds that before the Page time, the minimal choice for the island is the null (trivial) one, and therefore, up to that time, the new entropy is the same as the usual one which grows. In this case, $\frac{A(\partial I)}{4 G}$ is zero, whereas $S_{\text {new }}=S_{\text {usual. }}$. But, after the Page time, the choice of the island which minimizes the sum, among other choices of extremas, is the one just behind the horizon. In that case, $\frac{A(\partial I)}{4 G}$ becomes the dominant contributor, as in the other part, both of the entangled Hawking quanta (inside and outside the black hole) are included. Thus, this part contributes much less as the Hawking quanta are purified. This is an important point, which we will come back to while making connections to multipartite entanglement of purification. But, the

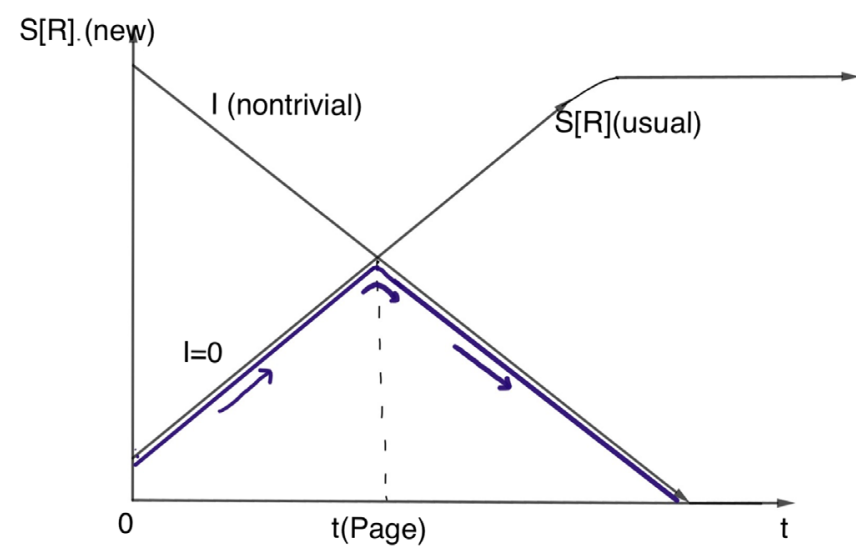

FIG. 5. Choice of islands before and after the Page time and Page curve.

dominant contributor $\left(\frac{A(\partial I)}{4 G}\right)$ decreases over time, which helps in the production of a Page curve (see Fig. 5).

\section{B. Multiboundary wormholes and a simple toy model}

In this subsection, we briefly discuss multiboundary wormholes in $\mathrm{AdS}_{3}$. Then we discuss the toy model introduced in [33], where the authors have shown that classical RT surfaces can also reproduce a Page curve in some situations and the aspects of the newly introduced islands can be intuitively understood from the perspective of quantum error correction [34,36,37].

Multiboundary wormholes occur when many boundary CFTs are connected by a wormhole. All of these different boundaries are independent of each other. The construction of multiboundary wormholes in $\mathrm{AdS}_{3}$ is a well-discussed topic and an active area of research in itself. In our usual understanding, multiboundary wormholes can be thought of as multiple exits created by quotienting $\mathrm{AdS}_{3}$ and by removing semicircles from a time slice of pure $\mathrm{AdS}_{3}$ by orientation-reversing isometries in the upper half plane. This defines the fundamental domain. Since in three spacetime dimensions, true dynamical degrees of freedom are lacking, only global topological data and boundary dynamics determine a classical saddle, implying that for smooth asymptotically $\mathrm{AdS}_{3}$, all geometries locally belong to the same universal class and are distinguished only by global features.

In AdS/CFT, this is related to the study of the $n$ fold tensor product of CFT states in different boundaries. For $n=2$, the resulting geometry is that of a BTZ which is dual to a thermofield double (TFD) state. Recently, Ref. [41] discussed these cases in detail. Figure 6 shows the way one creates two boundaries by removing two semicircles from a pure $\mathrm{AdS}_{3}$ slice at $t=0$ through a Killing vector that generates dilatation. The standard way of addressing dynamical questions in CFTs is the formalism known as Schwinger-Keldysh, which, in the context of holography, is 


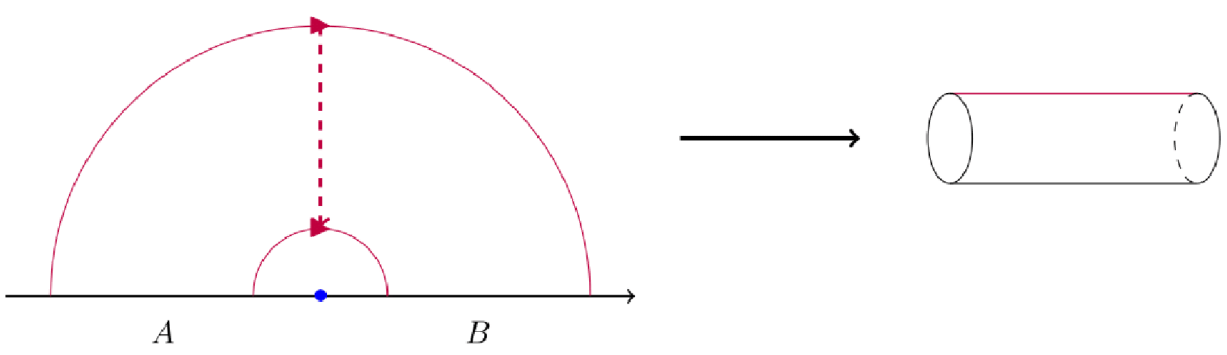

FIG. 6. Two-boundary case and horizon length, equivalent to EWCS for the bipartite system.

translated as considering multiboundary geometries in the Euclidean and Lorentzian signatures and gluing across a surface of zero extrinsic curvature (boundary-anchored geodesics). As a spacelike slice of $\mathrm{AdS}_{3}$ always maps onto the Poincare disk by stereographic projection, we start with a Poincare disk and take the quotient by a single hyperbolic isometry producing a Riemannian surface with constant negative curvature everywhere. This manifold is obtained if one cuts a strip bounded by geodesics anchored on the boundary out of the disk and glues it shut. This produces a time-symmetric slice at $t=0$ of a two-sided BTZ. Figure 6 is the fundamental domain of a two-sided BTZ, defined by removing two semicircles that are related through a dilatation. In reality, $A$ and $B$ are the two boundaries where two CFTs live. We will discuss these connections in detail in the next section.

Nevertheless, one can introduce more and more exits by removing more and more semicircles in an orientationreversing way (discussed in Appendix A) on one side of the smaller semicircle of Fig. 6. These removals simply correspond to quotienting by more and more isometries. Removing semicircles from the other side would mean introducing handles. ${ }^{1}$

A multiboundary wormhole can be understood as a diagram that resembles a pair of pants with more than two legs (see Fig. 7). In such a construction, all the different horizon lengths can be tuned or changed independently in terms of the parameter in the time slice of $\mathrm{AdS}_{3}$ through which the semicircles are removed. But for a two-boundary case, there is only one horizon, which the CFT sees, and there is only one parameter involved, which is the ratio of the radius of the semicircles in Fig. 6. Starting from $n>2$, an $n$-boundary wormhole would have parameters such that all the horizons can be made big or small independently using a nonoverlapping set of parameters.

Using the above-mentioned facts, the authors in [33] introduced a toy model, in which they considered a multiboundary wormhole where one of the horizons is much longer to start with than all other horizons. They begin with the $n=3$ case (see Fig. 8) (thus with the

\footnotetext{
${ }^{1}$ The introduction of a handle involves removing two semicircles from two sides of the lower semicircle of Fig. 6, but it also reduces the number of exits or horizons by one.
}
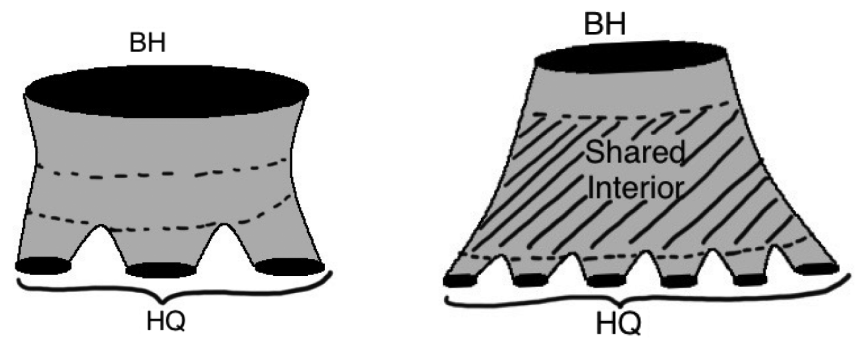

FIG. 7. Pantlike diagrams of MBW and the shared interior.

independence of parameters allowed from the start). They describe the boundary of the bigger horizon to be the evaporating black hole, whereas other black holes, which are much smaller in size and live in the smaller exits of the MBW picture, are considered to be the radiated Hawking quanta. With each emission of Hawking quanta, a new exit is created and the horizon length of the bigger evaporating black hole is reduced very little. The authors neglect the discussion regarding new topology (exits) created in such a process and any issue with bulk dynamics.

An important assumption the authors make is that the ADM energy is conserved during the evaporation process. Using this fact, one can define the smaller black holes (dual to emitted Hawking quanta in such a model) to be of a comparable length scale (the horizon length of all of them made almost equal, independently), which is much less than the evaporating one. But as along the process the ADM energy conservation is used and the evaporating black hole reduces in size, the HRT surfaces of the union of the disconnected Hawking quanta choose different horizons in different times. ${ }^{2}$ Primarily, the smaller choice of the HRT surface is the union of disconnected horizons of the smaller exits (Hawking quanta) whereas, at a later time, where the number of emitted quantas is much larger, the minimal HRT choice is the horizon of the evaporating black hole. This study produces a Page-like curve since, at later times, a different HRT surface is automatically more favorable and the region between the bigger and smaller

\footnotetext{
${ }^{2}$ In this picture, both the horizon corresponding to the bigger black hole and the union of the horizons of the smaller ones are homologous to both the bigger black hole and the union of smaller black holes or Hawking quanta.
} 

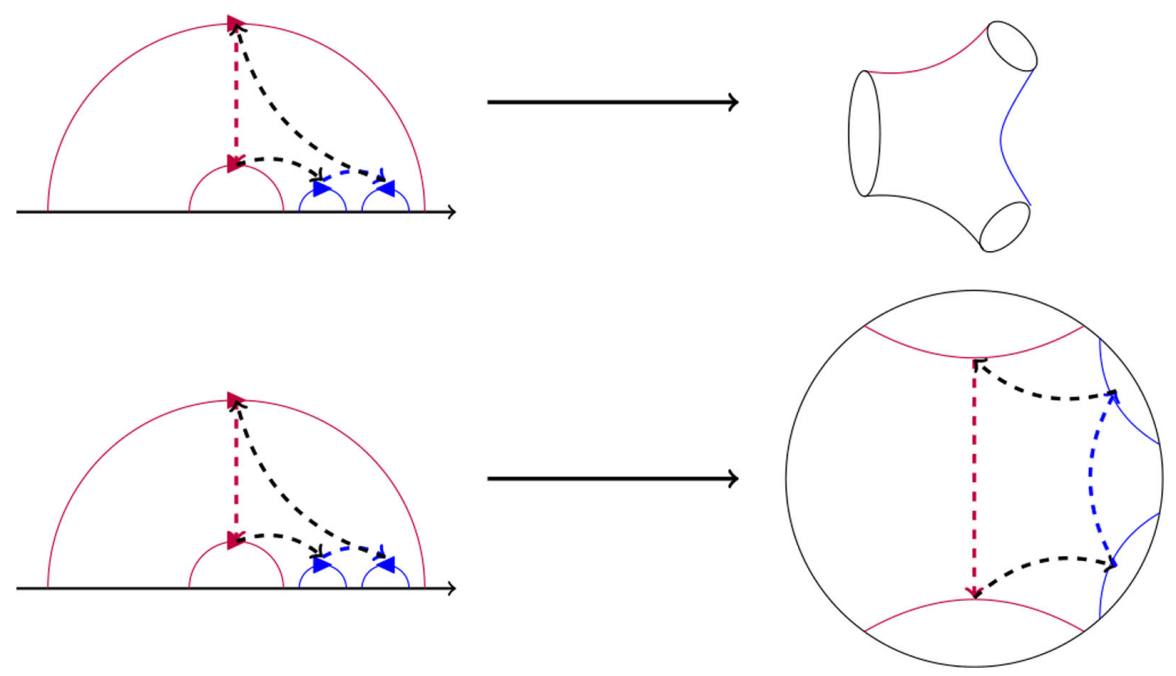

FIG. 8. Three-boundary Riemann surface as quotients of the two-boundary Riemann surface. The three-boundary surface is obtained by pinching one of the boundaries into two. The island is marked by the closed region shown by the dotted purple, black, blue, and black lines, respectively.

horizons is intuitively understood as the nontrivial island at the later times.

The relation between entropy and ADM energy in $\mathrm{AdS}_{3}$ is the following:

$$
S=2 \pi \sqrt{\frac{c E}{3}} .
$$

In three bulk spacetime dimensions, the area of the HRT surface is simply the length. Let us consider that the initial length of the horizon of the evaporating black hole is $L_{0}$, which decreases over time as it emits more small black holes with horizon lengths $\ell$. Using the relation between length (entanglement entropy) and ADM energy, we can show that, at any point of time, where $n$ smaller black holes have been emitted, the horizon length of the bigger black hole reduces in the following way,

$$
L(\mathrm{BH})=\sqrt{L_{0}^{2}-n \ell^{2}}
$$

whereas the union of the length of the smaller horizons scales like $L(\mathrm{HQ})=n \ell{ }^{3}$. For smaller values of $n, L_{\mathrm{HQ}}$ is the minimal choice, which grows over time as $n$ increases. The $L_{\mathrm{BH}}$ decreases as time moves forward. At a certain timescale $n \sim \frac{L_{0}}{\ell}, L_{\mathrm{BH}}$ and $L_{\mathrm{HQ}}$ become comparable, and after that, $L_{\mathrm{BH}}$ becomes the minimal HRT choice. This is how one can obtain a Page-like curve through this toy model (see Fig. 9).

The region between the chosen HRTs at different times (here the number of exits, $n$, is considered to be the analog

\footnotetext{
${ }^{3}$ Here, $L_{\mathrm{HQ}}$ corresponds to the length of the horizons of the smaller black holes that are analogs of Hawking quanta in the radiation.
}

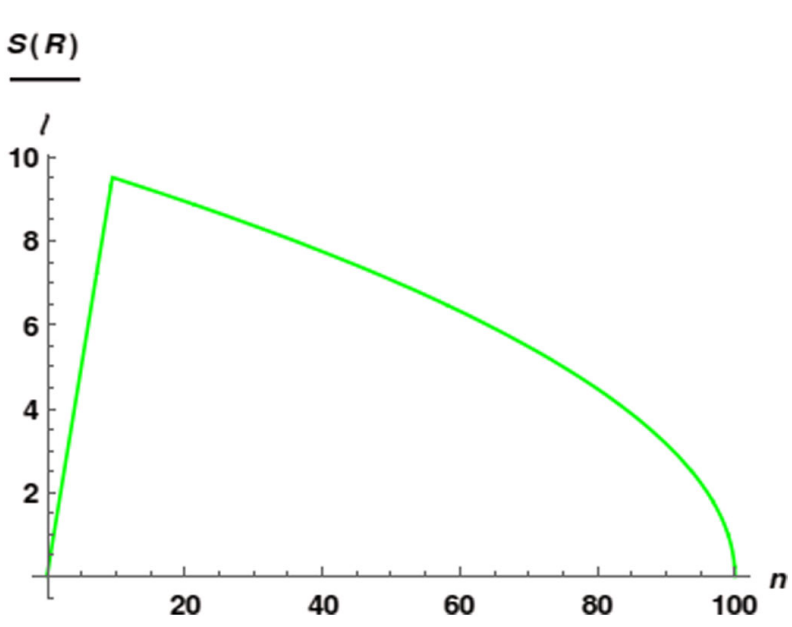

FIG. 9. Page curve from the MBW toy model.

of time) also has similarities with the shared interior that appears in the study of quantum error correction through the bulk reconstruction picture. Through this similarity, the authors in [33] provided a possible understanding of the islands, which comes from the full and restricted set of observables that can be reconstructed depending upon which surface is chosen. According to the authors, as the shared interior is not dual to any single boundary subregion, it appears as a quantum error-correcting region in the computation of entanglement entropy. In the next section, we discuss the two previous studies and make a few connections with them so that we can get a better understanding of what the toy model implies and how it can be connected to the study of multipartite entanglement of purification. 


\section{CONNECTIONS BETWEEN PURIFICATION AND MBW TOY MODEL}

In this section, we first make several connections between purification and the MBW toy model. Then, we discuss how the naive application of the island formula can lead to the earlier paradox. We also take lessons from other works that help us eliminate problems and better understand both scenarios.

\section{A. Connections to be drawn}

The two topics discussed in the previous sections have striking similarities, which have yet to be pointed out. We make the following observations and constructions, which help us understand the connections and give us a few important points that should be kept in mind while comparing the two scenarios.

(1) As we have already pointed out in the previous section, bipartite entanglement of purification, where the bipartition is made by choosing two disjoint but substantially larger subregions of a timeslice of pure $\mathrm{AdS}_{3}$, is very similar to the construction of a wormhole connecting two boundaries, where the boundaries exactly correspond to subregions $A$ and $B$ of the bipartite system.

To be more exact, in the case of two boundaries, one takes two boundary-anchored geodesics in the Poincare disk, the fundamental domain (corresponding to the HRT surfaces of the region $A \cup B$ not sharing any endpoints) and uses a unique isometry (dilatation to be precise) that defines a bijective map from points on one of the geodesics to the closest points on the other. In this map, one can identify points on the two geodesics periodically and glue them together. This isometry does not involve any fixed points in the strip between the two geodesics. ${ }^{4}$

(2) Another striking similarity is that the entanglement wedge cross section for a bipartite state is the only possible horizon length that one can compute in a twoboundary case. After the identification and gluing procedure is completed among the two boundaryanchored geodesics in the Poincare disk, one needs to specify that the two-sided BTZ is not two geodesics, but a single geodesic specifying the horizon length.

We can simply move on to other cases with more exits and compare the cases, where in one, we increase the number of exits in the MBW picture, and in the other, we introduce more disjoint subregions in one of the subregions $A$ or $B .^{5}$ But there is one subtlety involved that one should keep in mind while doing so. For $n \geq 3$, one needs to remove two semicircles to

\footnotetext{
${ }^{4}$ Multiboundary wormhole constructions can involve isometries, including pathologies like fixed points and closed timelike curves, in general. But these can be avoided by making suitable choices as mentioned in [41].

${ }^{5}$ If we introduce more subregions on both sides, that would mean introducing handles in the MBW picture. We avoid such scenarios for the time being.
}

introduce each new exit. Thus, after $n=2$, in the partitioning of the boundary, we have to introduce two disjoint subregions at each step in the purification picture, a combination of which will be equivalent to the newly constructed boundary.

In doing so, in each step, we should also keep decreasing the size of the other subsystem $A$ very slowly so that our picture agrees with the previously introduced toy model. We also introduce new subsystems in such a way that their contributions in multipartite EoP are much smaller initially with respect to the contribution of the bigger subregion $A$.

We can describe our newly defined subsystems in the following way so that they agree well with the boundaries that are defined in the MBW case. We take two disjoint boundary intervals connected to $A$ on two different sides and call them $B 1$ and $B 2$. We call their union partition $B$. Similarly, for more exits, we keep taking unions of the intervals connected to $B 1$ and $B 2$ on two sides and define them as a new partition (as shown in Fig. 10).

(3) Now, by looking at the two pictures, one can easily point out that the dotted lines in Figs. 7 and 8 are equivalent to each other. But, in multipartite EoP, we take their sum, whereas, in the MBW toy model, they are treated as two different sets that naturally provide one with a way to choose one of them as the HRT surface. If we look at the figures more carefully, it is not hard to see that the codimension-1 region enclosed by the multipartite entanglement wedge cross section is the shared interior (island) in the MBW picture. It is then obvious to define the multipartite entanglement wedge cross section as the boundary of the nontrivial island. We thus get an understanding of the boundary of the nontrivial island after the Page time in terms of entanglement of purification between the evaporating black holes and the radiation quantas (union of all other boundary subregions except $A$ ).

(4) An important point to note about the connections between the two scenarios is the correspondence between a geometric pure state and the multiboundary wormholes connecting the bigger and the smaller black holes. In the case of multipartite entanglement of purification, we consider the bulk HRT surfaces for the subsystems of the pure AdS along with the boundary subregions as a geometric pure state. ${ }^{6}$ The multiboundary wormhole connect-

\footnotetext{
${ }^{6}$ To be precise, the HRT surfaces of the multiboundary cases do not form a closed region by themselves. The shared interior is understood as the union of HRTs along with certain regions of the wormholes connecting different exits (see Appendix B for more details and Fig. 7). While considering a geometric pure state in the dual EoP picture, we make the choices $\Gamma_{\tilde{A} \tilde{B}}, \Gamma_{\tilde{B} \tilde{C}}$, and $\Gamma_{\tilde{A} \tilde{C}}$ in such a way that their combination forms a minimally closed curve (see Fig. 3) among all other choices. This choice is always the minimal choice for the boundary of the analog of the islands (see Fig. 13).
} 

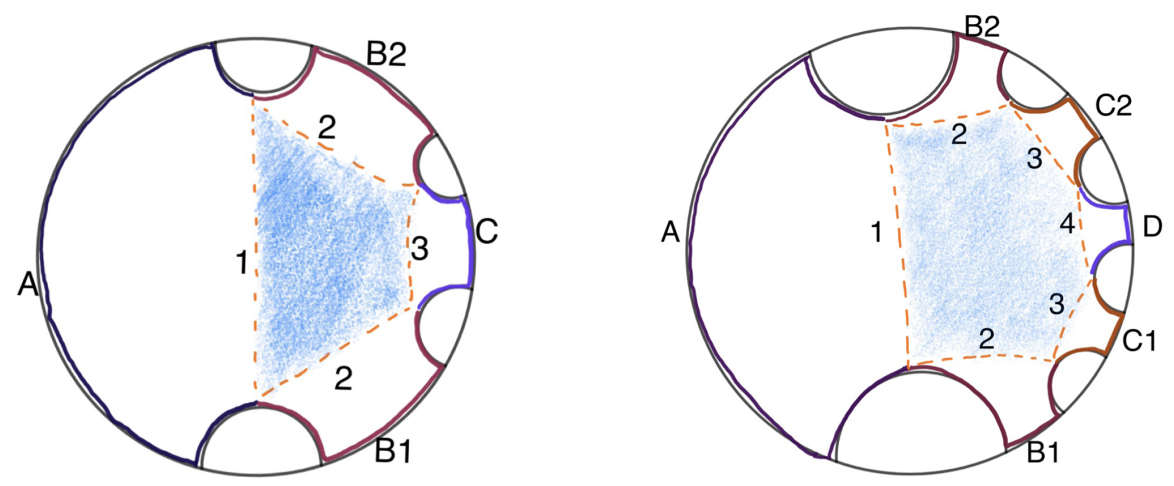

FIG. 10. Three- and four-boundary cases. The semicircles to be removed are all marked. Blue-shaded regions represent the shared interiors. These are the choices that minimize the boundary of the shared interior with respect to the corresponding geometric pure state.

ing CFTs at different exits acts as a machine to make the whole multipartite state (combination of multiple exits) a pure state. Making this connection helps us to understand the multiboundary wormhole, along with the exits as a geometric pure state for which we consider the HRT surfaces to compute the entanglement entropy. ${ }^{7}$

\section{B. Realization of overcounting through shared interior}

The previously mentioned comparisons and connections indeed support the connection between the islands and quantum error connection since multipartite EoP has connections to quantum error corrections as discussed in [42]. But as mentioned in the formula of the quantum extremal surface, if one computes the area (length in the case of $\mathrm{AdS}_{3}$ ) of the boundary of the island, it does not behave as we expect. This is because $A(\partial I)=\Delta_{W}$, and it consists of both the union of smaller horizons and of the bigger horizons. In addition, the length of the boundary of the nontrivial island would have the same properties as multipartite EoP, which we have already listed in Sec. II,

$$
A(\partial I)=\Delta_{W}=\sqrt{L_{0}^{2}-n \ell^{2}}+n \ell .
$$

Now, although the length of the bigger horizon keeps decreasing over time, the length of the combination of the smaller ones keeps increasing. Their sum still grows (see Fig. 11) until the black hole evaporates (here, this corresponds to the case where subregion $A$ becomes so small that

\footnotetext{
${ }^{7}$ Some of these connections were also drawn and discussed in [40]. Indeed, their discussion is more detailed. But the goal of that work was to advertise the entanglement entropies of multiboundary wormholes as equivalent to entanglement of purification of pure $\mathrm{AdS}$ and advocate that entanglement entropies are easier to compute than the EoP. Our study is a bit more safeguarded as we only make the connections by introducing a subregion in a single side of the bigger subregion, which does not introduce handles in the wormhole geometry.
}

the entanglement wedge [34] of the partitions simply becomes the union of the causal wedge of each of them).

Therefore, if one strictly assumes the shared interior to be the analog of the nontrivial island, its boundary area is ever-growing even after the Page time (shown in Fig. 11). Nevertheless, we prescribe the following resolution to the paradox. This is again the same paradox that the whole program began with. In the toy model, the authors try to realize the notion of the islands just through the classical HRT surfaces, neglecting the bulk entropy part (second term in QES equation), assuming that the lengths of the smaller horizons individually are enough to keep track of the bulk entanglement entropy associated with the smaller black holes.

The argument is not so unsatisfying once we take into account that the analogs of the Hawking quanta are small black holes in the MBW picture, which are classical geometric objects. But from the point of entanglement of purification, when we consider the whole multipartite EWCS, we include both the bigger black hole horizon and the smaller ones. If translated to the statements made in [33], this effectively means that we double count the bulk entropy of Hawking quanta in multipartite EWCS. Hence, once the island is included, in our calculations, the entanglement between the partner modes of the emitted quantas also contributes to the multipartite EoP. But, in fact, as the new HRT includes both partners, it is purified. Multipartite EoP is insensitive to this purification and overcounts it to make the entanglement of purification larger than what it should be.

The final resolution for the choice of multipartite entanglement wedge cross sections can be drawn from [43], in which, again, multipartite entanglement has been studied in detail. Drawing connections from that paper, we can resolve the problem in the following way. As one of the black holes is considered to be much bigger than the other ones, primarily all other horizon lengths can be considered as $\ell \rightarrow 0$. The reverse limit would be to take the smaller horizons $\ell$ as finite, whereas $L_{\mathrm{BH}} \rightarrow \infty$. In both of these limits, the combined state behaves like a bipartite state [43] 

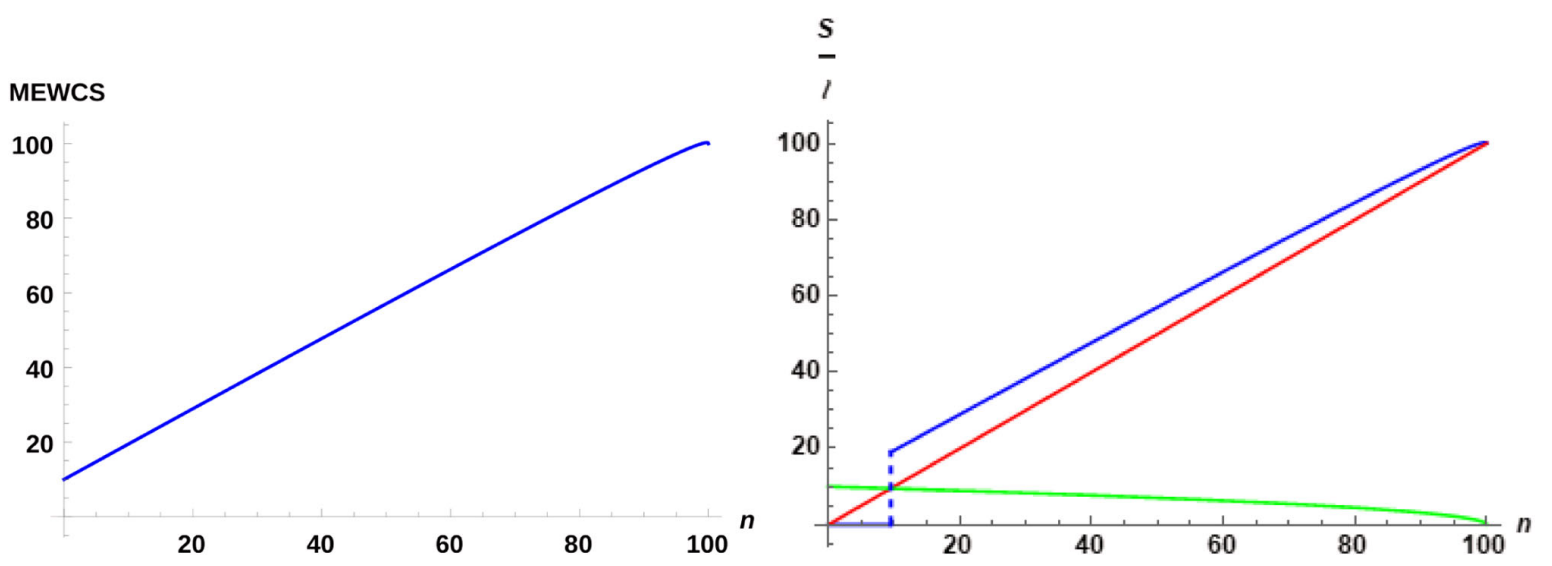

FIG. 11. Left panel: Growth of the multipartite EWCS (for the minimal choice). Right panel: Comparison between the primary (red) and later (green) choices of HRT with minimal island (blue) growth at different times.

between the bigger $\mathrm{BH}$ and the union of the smaller ones. In that situation, the multipartite case boils down to a simplified bipartite case, where the entanglement of purification reduces to the usual entanglement entropy. Therefore, one can simply choose either the union of the horizons of smaller black holes or the horizon of the bigger black hole as the HRT surface, depending on whichever is minimal at that time. But even in that scenario, if the newly entered shared interior is considered to be the analog of the nontrivial island, the growth of the boundary length of the island is paradoxical. The reason why this paradox arises only in the toy model is that, in this case, the analog of islands is connected to the earlier null island. On the other hand, in the actual case, the island is behind the black hole horizon and is disconnected from the trivial island choice before the Page time.

If we treat various smaller black holes differently, we would necessarily have to consider multipartite entanglement of purification. If we consider the bigger black hole as subsystem $A$, and $n$ smaller black holes as $B_{1}, B_{2}, \ldots$, and $B_{n}$, then multipartite EoP should be

$\Delta_{P}\left(A: B_{1}: \ldots . B_{n}\right)=\frac{1}{n} \min _{|\psi\rangle_{\text {pure }}} \sum_{i=1}^{n}\left(S_{A A^{\prime}}+S_{B_{i} B_{i}^{\prime}}\right)$.

In this scenario, all the smaller black holes are treated as a combination, and along with the bigger black hole, they form a pure state; we can take $A^{\prime}=B_{i}^{\prime}=\varnothing$, and therefore,

$\Delta_{(n+1) P}\left(A: B_{1}: \ldots .: B_{n}\right)=\Delta_{(2) P}(A: B)=\frac{1}{2}\left(S_{A}+S_{B}\right)$.

Note that it is necessary to consider the full state as a bipartite pure state. A multipartite pure state would not solve the problem. For example, if we consider that the combination of the big black hole and $n$ smaller black holes is an $(n+1)$-partite pure state, we would still have to apply property 2 of the multipartite EoP,
$\Delta_{P}\left(A: B_{1}: \ldots: B_{n}\right)=\sum_{i=1}^{n}\left(S_{A}+S_{B_{i}}\right)=\sqrt{L_{0}^{2}-n \ell^{2}}+n \ell$,

where for each small black hole, even after the Page time, $\ell$ would be the HRT surface for individual smaller black holes instead of $L_{\mathrm{BH}}$. Thus, the multipartite EoP would still give us the ever-growing entanglement. Therefore, the resolution appears only when in the limit of a very large number of very small black holes, and we take the union of the smaller black holes to be a single mixed state, which, along with the large black hole state, forms a bipartite pure state.

Through this study, we learn important lessons about the two scenarios, which we discuss in the following section.

\section{DISCUSSION AND OUTLOOK}

From the connections we made between multipartite entanglement of purification and multiboundary wormholes in $\mathrm{AdS}_{3}$, we can make the following points.

(1) First and foremost, the multipartite entanglement wedge cross section represents the boundary of the islands described in the toy model of the evaporating black hole. We believe that this would strengthen the possibility of building a concrete understanding of the islands as well as purification in several different ways, e.g., quantum error correction, entanglement negativity, and many more. Precisely, in the large $n$ limit, i.e., where the number of smaller exits is big, multipartite EoP and the shared island match completely. In the multiboundary wormhole picture, the shared island is the region behind all the horizons present (which is also the case in the original works discussing the actual model), and therefore it falls in the region known as the entanglement shadow. Our work suggests that, through the multiboundary wormhole construction, the entanglement shadow 
can have a description through the multipartite EoP of subregions in a vacuum $\mathrm{AdS}_{3}$ slice.

(2) The reproduction of the Page curve helps describe an evaporating black hole as a unitary system since the Page curve is typically found in systems that evolve unitarily over time. Now, given the appearance of islands, or rather quantum error connection, ensures that the unitarity of the black hole evaporation process is recovered, one would hope that they are related. The natural way to somewhat realize the connection is of course through the purification of the Hawking quanta after the Page time. This results in the appearance of nontrivial islands. In our study, we use the ideas of purification regularly, which gives rise to the multipartite EWCS, and the area enclosed is understood as the nontrivial island (quantum error correction). In other studies, for example, in [42], the authors have explored connections between entanglement of purification and quantum error correction. But, it would be really interesting to understand such a connection as a triangular relation where the three vertices of the triangle correspond to unitarity, purification, and quantum error correction. ${ }^{8}$ Regarding the line connecting unitarity to purification, a realization is the fact that a reduced density matrix (from which the purification is typically done) is derived by tracing out degrees of freedom from the initial pure state,

$$
\rho_{\text {red }}=\operatorname{Tr}_{(\text {pure-red })}\left[\rho_{\text {pure }}\right]
$$

This tracing-out is a nonunitary operation. Therefore, the reduced density matrix indeed carries the effect of a nonunitary operation. Hence, it is not beyond our expectations that, to get back the unitarity completely, one needs to apply purification to the reduced mixed state. This can be pursued in more detail to get a better understanding of the above-mentioned triangle.

(3) Although the islands can be intuitively understood as the shared interior, the length associated with the boundary of such a shared interior leads to a problem in overcounting, due to which the entropy associated with the shared interior does not follow the Page curve (since the growth of the boundary of the nontrivial island after the Page time persists, as it includes the previously chosen HRT surfaces as well). To be precise, in the toy model, it is assumed that the RT surfaces take care of the bulk entropy between the fields that live on different sides of the HRT. But once the new choice of HRT is made, it

\footnotetext{
${ }^{8}$ We thank Arnab Kundu for pointing out this interesting future direction.
}

contains both the partner modes. For modes without the partner modes inside the new HRT, their bulk entanglement with their partners is again satisfied by the new HRT. But, if one computes the sum of the length of the shared interior simply by considering it to be the boundary of the island, one again counts the bulk entanglement between the modes, which have already been purified due to the choice of the new HRT. Let us call the shared interior SI. Then,

$$
L(\partial(\mathrm{SI}))=L_{\mathrm{BH}}+L_{\mathrm{HQ}}
$$

The SI only comes into the picture after the choice of the nontrivial island is minimal. Starting from that point, the boundary of SI also includes $L_{\mathrm{HQ}}$, which has bulk entanglement between partner modes of the two sides of the previous HRT choice, and this is how the overcounting can again come into the picture.

In [33], the authors introduce a second model involving handles and pairs of TFD states of the baby universe and the radiation states to understand the previous overcounting that led to the information paradox in the first place. We see here that even without introducing a new model, one can get the overcounting from the very first model by naively following the formula of the quantum extremal surface to include the whole length of the boundary of the nontrivial island and return to the earlier paradox.

However, taking the intuitive understanding of islands too literally might lead to several problems. In this particular toy model, it is necessary to compute the lengths of the chosen HRTs only at any point in time. One does not need to include the bulk entanglement, but it is also wrong to consider the remaining part of the quantum extremal surface formula in terms of the island. This subtlety might also capture important insights in making a connection between islands and QEC more concrete since QEC is well studied in the literature of EoP [42].

(4) For the problem of multipartite EoP, one can make simple calculations that show how the multipartite EoP grows over time and how different parts of it contribute to the Page curve in the multiboundary wormhole model of black hole evaporation in $\mathrm{AdS}_{3}$. But this brings up another question that needs further understanding and study: whether in multipartite EoP, there is any overcounting taking place that one needs to be aware of. Since we consider the union of smaller black holes as our radiation state, a possible resolution in the purification side is to simply consider that in the given limits (one black hole much larger than all the other ones), the total state 
behaves as a bipartite state instead of a multipartite state, and the entanglement of purification reduces to the usual entanglement entropy,

$$
\Delta_{W}\left(A: B_{1}: B_{2}: \ldots: B_{n}\right) \rightarrow E_{P}(A: B)=S_{A}=S_{B},
$$

where $A$ is the bigger black hole and $B$ is the union of the smaller black holes $\left(B_{1}, B_{2}, \ldots, B_{n}\right)$. Specifying $S_{A}=S_{B}$ at different times depends on which part of $\Delta_{W}$ is the minimal choice. For example, we can divide $\Delta_{W}$ into two parts, one coming from the EWCS of two-boundary wormholes $\left(\Delta_{W, 1}=\sqrt{L_{0}^{2}-n \ell^{2}}\right)$ and the other coming from the EWCS of the unions of the smaller boundaries $\left(\Delta_{W, 2}=n \ell\right)$. At all times, we can write

$$
\Delta_{W}=\Delta_{W, 1}+\Delta_{W, 2},
$$

and at each time (for $n>2$ ), in the limit where we consider the multipartite pure state as a bipartite one,

$$
\begin{aligned}
E_{P}(A: B) & =S_{A}=S_{B}=\min \left(\Delta_{W, 1}, \Delta_{W, 2}\right) \\
& =\min \left(\sqrt{L_{0}^{2}-n \ell^{2}}, n \ell\right)
\end{aligned}
$$

This is a justifiable assumption since, ultimately, we are concerned with the entanglement between the radiation state (union of smaller black holes) and the evaporating black hole state. Therefore, it is not so unexpected that the initially multipartite situation reduces to a simpler bipartite one. A detailed fieldtheoretic study similar to [43] in terms of purification would be able to shed more light on the necessity of this consideration. This is an ongoing problem being studied. Also, it would be interesting to consider different exits of a multiboundary case differently and study how multipartite EoP behaves.

(5) At this point, it is also important to note that how a multipartite EoP is reduced to a bipartite case is very similar and pictorially the same as choosing just the area of the HRT instead of choosing the area of the nontrivial island in this toy model. In both cases, one would encounter the information paradox, had the alternate choice been made.

Recently, Ref. [44] studied the multiboundary wormholes and purification from a different perspective. The model introduced in [44] works with end-of-world branes, and the multiboundary wormhole appears in the auxiliary system introduced for the purification. That work considers something termed as "inception geometry" to propose an extremal surface through which the nontrivial islands can again be marked. They argue that there are some regions behind the horizon which can only be found if the Hawking radiation is considered as a union of different subsystems of the radiation. They call such an event a quantum/geometric secret sharing. In our discussion, we find that it is necessary to finally consider the system of big and smaller black holes as a bipartite pure state to make sense of the Page curve. But nevertheless, it is absolutely necessary to model numerous smaller black holes (subsystem Hawking radiation) to get the analog and intuitive understanding of islands. Had we just considered a bipartite pure state, i.e., a two-boundary wormhole, we would never be able to get the shared interior which appears after the Page time. Note that in this case, there is only one choice for the EWCS as well as the HRT. Therefore, our discussion, in a way, also addresses the necessity of modeling the radiation as a union of subsystems as discussed in [44]. In light of such findings, we prefer to make the following statement: Although multipartite purification in the multiboundary wormhole toy model returns the overcounting once the boundary of the island is computed, it is absolutely necessary to primarily have the multipartite nature in the modeling of the radiation states to have a realization of the islands in the toy model. To resolve the overcounting issue, we nevertheless need to review the model as the bipartite one and choose the minimal one among the two parts of the multipartite EWCS as the entanglement entropy of the bipartite pure state.

Along with these lessons of how the two sides can feed each other with valuable new information, several future problems remain open. The realization of both the multiboundary wormhole and multipartite entanglement of purification in higher space-time dimensions would be an interesting direction to pursue. A similar connection between multipartite EoP and MBW was made earlier in [40] but with a different goal. ${ }^{9}$ But the authors missed the subtlety of connecting subregions to boundaries when a handle is also involved in the MBW picture. It would be interesting to investigate whether such a description of islands in terms of multipartite EoP persists in higher dimensions and situations involving handles in the wormhole geometries. This would, in principle, mean that one will be able to make stronger statements about the connection between islands and quantum error correction. It is also important to note that since QEC relates entanglement wedge cross sections to entanglement negativity, the connections between EoP and islands are also worth studying. Since entanglement wedge cross sections are also argued to pave the way towards a better understanding of full tensor networks in AdS/CFT, as advocated in $[45,46]$, this can also be a potential candidate in providing us with a parallel understanding of the islands in terms of the tensor networks.

We believe this study will open up several directions that can be explored. These future studies can potentially

\footnotetext{
${ }^{9}$ We thank Aidan Chatwin-Davies for bringing this to our attention.
} 
connect many of the recent exciting topics and strengthen our understanding of them.

\section{ACKNOWLEDGMENTS}

It is a pleasure to thank Shibaji Roy, Arnab Kundu, Harvendra Singh, and other members of the String Group in SINP for many useful discussions. We would also like to acknowledge Shibaji Roy, Arnab Kundu, Arpan Bhattacharyya, and Avik Banerjee for useful comments and suggestions on the earlier draft of this work. Finally, we would like to thank the Department of Atomic Energy (DAE), Government of India for the funding.

\section{APPENDIX A: MORE ON MULTIBOUNDARY WORMHOLE CONSTRUCTION}

As mentioned in the main text, there are different methods for constructing multiboundary wormholes that have been discovered in other works. In [41], a global three-boundary wormhole construction was discussed. The idea is to use the Killing vectors of $\mathrm{AdS}_{3}$ in the $t=0$ slice in Poincare coordinates. The exponentiated Killing vectors are used as isometries in the identity component of $\mathrm{SO}(2,2)$. The Killing vectors form an so(2,2) algebra. In the case of a $t=0$ slice, the fixed points lie outside the fundamental domain, and three of the six Killing vectors are zero already.

The Poincare metric of $\mathrm{AdS}_{3}$ is written in the following way,

$$
d s^{2}=\frac{\ell^{2}}{p^{2}}\left(-d t^{2}+d x^{2}+d p^{2}\right),
$$

and the Killing vectors in the $t=0$ slice are of the following form,

$$
\begin{gathered}
\left.J_{01}\right|_{t=0}=0 \\
\left.J_{02}\right|_{t=0}=0 \\
\left.J_{03}\right|_{t=0}=0 \\
\left.J_{12}\right|_{t=0}=-x \partial_{x}-p \partial_{p} \\
\left.J_{13}\right|_{t=0}=\left(\frac{\ell^{2}-x^{2}+p^{2}}{2 \ell}\right) \partial_{x}-\frac{x p}{\ell} \partial_{p} \\
\left.J_{23}\right|_{t=0}=\left(\frac{-\ell^{2}-x^{2}+p^{2}}{2 \ell}\right) \partial_{x}-\frac{x p}{\ell} \partial_{p} .
\end{gathered}
$$

A basis change of $J_{13}$ and $J_{23}$ simplifies the action of these Killing vectors, or rather the isometries (the exponentiated version) on the complex upper half plane defined by

$$
z=x+i p, \quad \bar{z}=x-i p .
$$

In the new basis $J_{12}$ acts as dilatation, $\left(J_{13}-J_{23}\right)$ as translation, and $\left(J_{13}-J_{23}\right)$ as special conformal transformation. These and the inversion can provide all the necessary transformations of a semicircle on the upper half plane to generate multiboundary wormholes with an arbitrary number of boundaries $(n)$ and genus $(h)$. All connected, hyperbolic Riemann surfaces of genus $h$ and boundaries $n$ are denoted by $(n, h)$, and for a particular value of $n$ and $h$, the moduli space is known as the Teichmüller space. It parametrically presents the number of geometrical parameters needed to construct a multiboundary wormhole. For a $(2,0)$ wormhole, the number is just one (the horizon length of the two-sided BTZ). Otherwise, it consists of $3 h-3+2 n$ minimal geodesics and $3 h-3+n$ twist angles.

For a $(3,0)$ wormhole, the number of parameters is thus just three, all of which correspond to the horizon lengths. These three horizon lengths $L_{1}, L_{2}, L_{3}$ are functions of a mutually exclusive set of parameters (as found in [41]), and hence their lengths can be tuned independently in the construction. In order for our corresponding model to realize this from the subregion point of view, the same thing can be done by identifying the semicircles removed as the boundary subregions, and the entanglement of purification is then considered only for the regions in the remaining part of the boundary subregions, which correspond to the actual boundaries in the multiboundary wormhole construction. The boundary-anchored geodesics play the role of the gluing surfaces (as discussed in Sec. III and Figs. 6 and 7 of [40]).

The fundamental domain in the upper half plane would simply correspond to a decompactified Poincare disc picture for the subregions, and the semicircles in Fig. 12 are the analogs of the boundary-anchored geodesics, again in a decompactified Poincare disc picture.

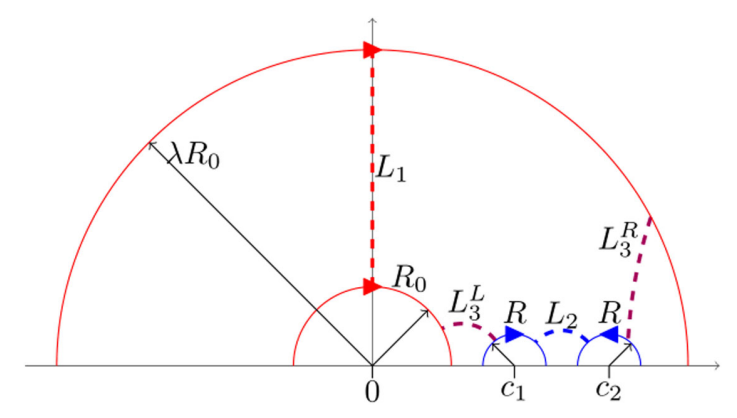

FIG. 12. Fundamental domain of the three-boundary Riemann surface $(3,0)$. The colored dashed lines $L_{1,2,3}$ are the minimal periodic geodesics, whose lengths are the physical parameters of the system. The variables $\lambda, R_{0}, R, c_{1}$, and $c_{2}$ represent parameters for the picture, which are related to different Killing vectors that transform the semicircles through isometries. 


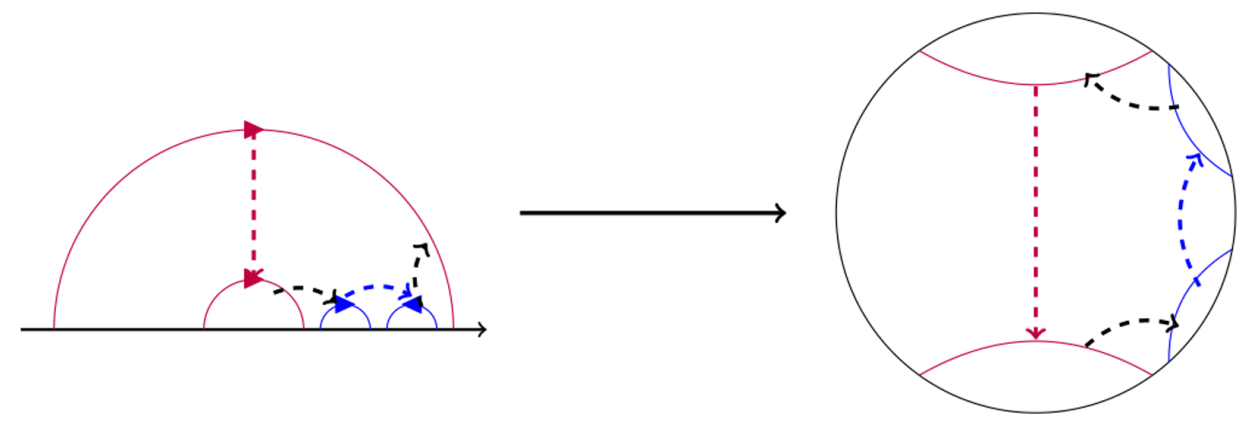

FIG. 13. Three-boundary Riemann surface as quotients of the two-boundary Riemann surface. The left diagram shows the usual horizon choices in the upper half plane where the boundary of the island consists of the dotted lines (horizons) and the union of the solid lines between them. The right diagram shows the same region in the Poincare disk.

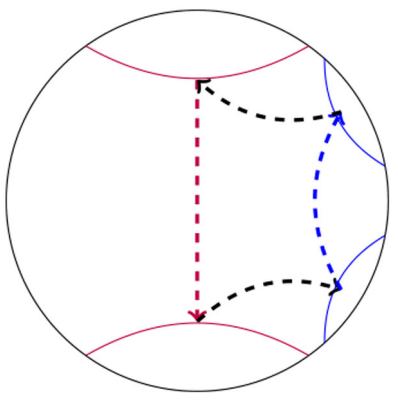

FIG. 14. Multipartite EoP where the bulk minimal geodesics form a closed region. In the case of a large number of exits, Fig. 13 matches with this kind of closed bulk island.

\section{APPENDIX B: LIMITATIONS AND CLARIFICATIONS}

In this section, we list the precise limitations of our proposal.

(1) The horizons in the multiboundary wormholes by themselves do not form a closed curve in the bulk, in general, as shown in Fig. 13. They also include part of the boundary-anchored geodesics. But, in the case of multipartite EoP, it is conjectured using the surface state correspondence that it is indeed a closed region (as shown in Fig. 14) in the bulk and does not involve any part from the boundary-anchored geodesics except the points where different entanglement wedge cross sections meet each other. Hence, the two pictures do not completely satisfy all the similarities in the most general situation. But, if we keep increasing the number of smaller exits, in the Poincare disk, we can easily see the part of the island that comes from intervals of the boundary-anchored geodesics and not the horizons keeps decreasing. Thus, in the large $n$ limit (which is also the subject of interest in our model at later times), the parts of the island coming from the boundary-anchored geodesic intervals tend to zero, and the two pictures exactly correspond to each other in this limit.

(2) In [40], the authors compared EoP and MBW horizons to point out that they correspond physically, but one is easier to compute, in general, than the other. But in our study, we find that the multipartite EoP, which is the analogue of the island, is not so easy to compute explicitly, in general. Physically, the island region also corresponds to the region behind all of the horizons and, thus, constitutes the entanglement shadow. Although it is hard to compute explicitly, which is a limitation, it provides reassuring physical hints that entanglement behind the horizon can be computed from the outside.
[1] S. W. Hawking, Black holes and thermodynamics, Phys. Rev. D 13, 191 (1976).

[2] L. Bombelli, R. K. Koul, J. Lee, and R. D. Sorkin, A quantum source of entropy for black holes, Phys. Rev. D 34, 373 (1986).

[3] M. Srednicki, Entropy and Area, Phys. Rev. Lett. 71, 666 (1993).
[4] C. Holzhey, F. Larsen, and F. Wilczek, Geometric and renormalized entropy in Conformal Field Theory, Nucl. Phys. B424, 443 (1994).

[5] M. Rangamani and T. Takayanagi, Holographic Entanglement Entropy, Lect. Notes Phys. 931, 1 (2017).

[6] P. Calabrese and J. L. Cardy, Entanglement Entropy and Quantum Field Theory, J. Stat. Mech. (2004) P06002. 
[7] J. Eisert, M. Cramer, and M. B. Plenio, Area laws for the Entanglement Entropy—A review, Rev. Mod. Phys. 82, 277 (2010).

[8] T. Nishioka, S. Ryu, and T. Takayanagi, Holographic Entanglement Entropy: An overview, J. Phys. A 42, 504008 (2009).

[9] T. Takayanagi, Entanglement Entropy from a holographic viewpoint, Classical Quantum Gravity 29, 153001 (2012).

[10] S. Ryu and T. Takayanagi, Holographic Derivation of Entanglement Entropy from Antide Sitter Space/Conformal Field Theory Correspondence, Phys. Rev. Lett. 96, 181602 (2006).

[11] D. L. Jafferis, A. Lewkowycz, J. Maldacena, and S. J. Suh, Relative entropy equals bulk relative entropy, J. High Energy Phys. 06 (2016) 004.

[12] A. Lewkowycz and J. Maldacena, Generalized gravitational entropy, J. High Energy Phys. 08 (2013) 090.

[13] T. Takayanagi and K. Umemoto, Entanglement of purification through holographic duality, Nat. Phys. 14, 573 (2018).

[14] P. Caputa, M. Miyaji, T. Takayanagi, and K. Umemoto, Holographic Entanglement of Purification from Conformal Field Theories, Phys. Rev. Lett. 122, 111601 (2019).

[15] A. Bhattacharyya, A. Jahn, T. Takayanagi, and K. Umemoto, Entanglement of Purification in Many Body Systems and Symmetry Breaking, Phys. Rev. Lett. 122, 201601 (2019).

[16] A. Bhattacharyya, T. Takayanagi, and K. Umemoto, Entanglement of purification in free scalar field theories, J. High Energy Phys. 04 (2018) 132.

[17] C. Akers and P. Rath, Entanglement wedge cross sections require tripartite entanglement, J. High Energy Phys. 04 (2020) 208.

[18] N. Bao and I. F. Halpern, Conditional and multipartite entanglements of purification and holography, Phys. Rev. D 99, 046010 (2019).

[19] K. Umemoto and Y. Zhou, Entanglement of purification for multipartite states and its holographic dual, J. High Energy Phys. 10 (2018) 152.

[20] N. Bao and I. F. Halpern, Holographic inequalities and entanglement of purification, J. High Energy Phys. 03 (2018) 006.

[21] Y. Kusuki and K. Tamaoka, Dynamics of entanglement wedge cross section from conformal field theories, arXiv:1907.06646.

[22] K. Umemoto, Quantum and classical correlations inside the entanglement wedge, Phys. Rev. D 100, 126021 (2019).

[23] S. Dutta and T. Faulkner, A canonical purification for the entanglement wedge cross-section, arXiv:1905.00577.

[24] N. Bao and N. Cheng, Multipartite reflected entropy, J. High Energy Phys. 10 (2019) 102.

[25] J. Chu, R. Qi, and Y. Zhou, Generalizations of reflected entropy and the holographic dual, J. High Energy Phys. 03 (2020) 151.

[26] M. Moosa, Time dependence of reflected entropy in Conformal Field Theory, J. High Energy Phys. 05 (2020) 082 .
[27] H.-S. Jeong, K.-Y. Kim, and M. Nishida, Reflected entropy and entanglement wedge cross section with the first order correction, J. High Energy Phys. 12 (2019) 170.

[28] Y. Kusuki and K. Tamaoka, Entanglement wedge cross section from CFT: Dynamics of local operator quench, J. High Energy Phys. 02 (2020) 017.

[29] A. Almheiri, R. Mahajan, and J. Maldacena, Islands outside the horizon, arXiv:1910.11077.

[30] A. Almheiri, T. Hartman, J. Maldacena, E. Shaghoulian, and A. Tajdini, Replica wormholes and the entropy of Hawking radiation, J. High Energy Phys. 05 (2020) 013.

[31] A. Almheiri, R. Mahajan, J. Maldacena, and Y. Zhao, The page curve of hawking radiation from semiclassical geometry, J. High Energy Phys. 03 (2020) 149.

[32] A. Almheiri, N. Engelhardt, D. Marolf, and H. Maxfield, The entropy of bulk quantum fields and the entanglement wedge of an evaporating black hole, J. High Energy Phys. 12 (2019) 063.

[33] C. Akers, N. Engelhardt, and D. Harlow, Simple holographic models of black hole evaporation, arXiv: 1910.00972.

[34] D. Harlow, TASI lectures on the emergence of bulk physics in AdS/CFT, Proc. Sci. TASI2017 (2018) 002.

[35] C. Akers, N. Engelhardt, G. Penington, and M. Usatyuk, Quantum maximin surfaces, arXiv:1912.02799.

[36] F. Pastawski, B. Yoshida, D. Harlow, and J. Preskill, Holographic quantum error-correcting codes: Toy models for the bulk/boundary correspondence, J. High Energy Phys. 06 (2015) 149.

[37] X. Dong, D. Harlow, and A. C. Wall, Reconstruction of Bulk Operators within the Entanglement Wedge in GaugeGravity Duality, Phys. Rev. Lett. 117, 021601 (2016).

[38] N. Jokela and A. Pnni, Notes on entanglement wedge cross sections, J. High Energy Phys. 07 (2019) 087.

[39] K. Tamaoka, Entanglement Wedge Cross Section from the Dual Density Matrix, Phys. Rev. Lett. 122, 141601 (2019).

[40] N. Bao, A. Chatwin-Davies, and G. N. Remmen, Entanglement of purification and multiboundary wormhole geometries, J. High Energy Phys. 02 (2019) 110.

[41] E. Caceres, A. Kundu, A. K. Patra, and S. Shashi, A killing vector treatment of multiboundary wormholes, J. High Energy Phys. 02 (2020) 149.

[42] J. Kudler-Flam and S. Ryu, Entanglement negativity and minimal entanglement wedge cross sections in holographic theories, Phys. Rev. D 99, 106014 (2019).

[43] V. Balasubramanian, P. Hayden, A. Maloney, D. Marolf, and S. F. Ross, Multiboundary wormholes and holographic entanglement, Classical Quantum Gravity 31, 185015 (2014).

[44] V. Balasubramanian, A. Kar, O. Parrikar, G. Srosi, and T. Ugajin, Geometric secret sharing in a model of Hawking radiation, arXiv:2003.05448.

[45] P. Nguyen, T. Devakul, M. G. Halbasch, M. P. Zaletel, and B. Swingle, Entanglement of purification: From spin chains to holography, J. High Energy Phys. 01 (2018) 098.

[46] N. Bao, G. Penington, J. Sorce, and A. C. Wall, Holographic tensor networks in full AdS/CFT, arXiv:1902.10157. 